\title{
Impact of third-order dispersion on the evolution of parabolic pulses
}

\author{
Sonia Boscolo and Brandon G. Bale \\ Photonics Research Group, School of Engineering and Applied Science, \\ Aston University, Birmingham B4 7ET, UK
}

\begin{abstract}
We present a perturbation analysis that describes the effect of third-order dispersion on the similariton pulse solution of the nonlinear Schrödinger equation in a fibre gain medium. The theoretical model predicts with sufficient accuracy the pulse structural changes induced, which are observed through direct numerical simulations.
\end{abstract}

Keywords: Nonlinear fibre optics, nonlinear wave propagation, self-similarity

\section{INTRODUCTION}

Intense optical pulses with an initial parabolic intensity profile retain their shape and acquire a linear frequency chirp upon propagation in optical fibres with normal second-order (group-velocity) dispersion (SOD). ${ }^{1,2}$ In the additional presence of linear amplification, parabolic pulses represent a common asymptotic state (attractor) for arbitrary initial conditions. ${ }^{3-7}$ Their intrinsic resistance to optical wave breaking and self-similar amplification behaviour makes parabolic pulses or similaritons very attractive for achieving high-energy, ultrashort optical pulses in fibre lasers operating in the normal dispersion regime. ${ }^{8}$ The normal SOD effectively linearizes the accumulated phase of the pulse allowing for the spectral bandwidth to increase without destabilizing the pulse. ${ }^{1}$ Along with fibre amplifiers and lasers, stable similariton pulses can also be generated in passive optical fibres provided a suitable tapering of the SOD profile is introduced. ${ }^{9}$ This approach is based on the observation that a longitudinal decrease of the normal dispersion is formally equivalent to linear gain. Analytically, self-similar parabolic pulses can be found as asymptotic, approximate solutions of the nonlinear Schrödinger (NLS) equation with gain in the semi-classical (large-amplitude or small-dispersion) limit. ${ }^{3,6}$

To date, most of the theoretical descriptions of the self-similar pulse shaping regime have been based on the assumption that only low-order nonlinear and dispersion effects impact pulse evolution. In fact, several novel features in the similariton regime have been observed due to the presence of third-order dispersion (TOD) in fibre amplifiers and mode-locked lasers. ${ }^{10-13}$ Further, in passive dispersion-decreasing fibers the SOD decreases asymptotically to zero making the relative contribution of higher-order dispersion significant. The inherent TOD in the fibre can severely affect similariton pulse propagation performance and lead to optical shock-type instabilities. ${ }^{14,15}$ As a result of the TOD, the pulse shape experiences an asymmetric temporal development with the peak shifted towards the edges of the pulse, which is eventually halted by pulse break-up. The acceleration of the center of mass of the pulse also results in an asymmetric spectrum.

In this paper, we summarize results in Ref. 16. We describe the structural changes induced by TOD on the similariton pulse solution of the NLS equation with constant linear gain. Our approach relies on the observation that there is a natural small parameter in the problem of self-similar parabolic pulse evolution: the ratio of the pulse temporal width to the peak amplitude. Analytic expressions are provided for the perturbations of the pulse amplitude and phase, and are shown to be approximated by cubic polynomials in the temporal variable. Further, the pulse skewness is calculated, and the analytic results are confirmed by extensive NLS numerical simulations.

Further author information: (Send correspondence to S.B.)

S.B.: E-mail: s.a.boscolo@aston.ac.uk, Telephone: +44 (0)121 2043495

B.G.B.: E-mail: b.bale@aston.ac.uk

Nonlinear Optics and Applications IV, edited by Benjamin J. Eggleton, Alexander Luis Gaeta, Neil G. R. Broderick Proc. of SPIE Vol. 7728, 77280F - (c) 2010 SPIE · CCC code: 0277-786X/10/\$18 · doi: 10.1117/12.854782 


\section{BASIC EQUATIONS}

The evolution of the field envelope $U$ of optical pulses in a nonlinear optical fibre with a constant gain profile and in the presence of TOD can be described by the extended amplified NLS equation ${ }^{17}$

$$
i U_{z}-\frac{\beta_{2}}{2} U_{t t}+\sigma|U|^{2} U-i \frac{g}{2} U=i \frac{\beta_{3}}{6} U_{t t t},
$$

where $\beta_{2}, \beta_{3}$ and $\sigma$ are the respective SOD, TOD and Kerr nonlinearity coefficients of the fibre, and $g$ is the constant gain along the fibre. Here we have neglected higher order effects in the gain such as gain saturation and gain bandwidth. ${ }^{3}$ We seek a solution of Eq. (1) of the form: $U(z, t)=a(z) f(\eta, \xi) \exp \left[i C(z) t^{2}\right]$, where new self-similar variables are introduced as $\xi=t / \tau(z)$ and $\mathrm{d} \eta / \mathrm{d} z=\sigma a^{2}(z){ }^{6}{ }^{6}$ Here, $a(z)$ describes the evolution of the pulse peak amplitude, $\tau(z)$ is a characteristic width, $C(z)$ is the chirp parameter, and $f(\eta, \xi)$ is a normalized, dimensionless function that describes the evolution of the pulse temporal profile and contributions to the peak amplitude and phase through its $\xi$ and $\eta$ dependencies, respectively. Inserting this into (1), we obtain coupled equations for $a, \tau$ and $C$, along with the governing equation for the structural function $f$

$$
i f_{\eta}-\epsilon^{2} \frac{\beta_{2}}{2 \sigma \tau^{4}} f_{\xi \xi}+\left(|f|^{2}+\lambda \xi^{2}\right) f=i \epsilon^{2} \frac{\beta_{3}}{6 \sigma}\left[\frac{1}{\tau^{5}} f_{\xi \xi \xi}+\frac{6 i C}{\tau^{3}}\left(\xi f_{\xi}\right)_{\xi}-\frac{12 C^{2}}{\tau} \xi(\xi f)_{\xi}-8 i C^{3} \tau \xi^{3} f\right] .
$$

Here $\lambda$ is an arbitrary separation parameter that depends on the pulse power, and the parameter $\epsilon \equiv \tau(z) / a(z)$. The solutions of the $(a, \tau, C)$ coupled system ${ }^{6}$ show that $\epsilon$ is a natural small parameter in this problem:

$$
\epsilon \equiv \frac{\tau(z)}{a(z)}=\frac{\tau_{0}}{a_{0}}=6 \sqrt{\sigma \beta_{2} /\left(2 g^{2}\right)} \ll 1 .
$$

Here $a_{0}$ and $\tau_{0}$ are the initial pulse amplitude and width. For example, for realistic rare-earth-doped fibre parameters: $\beta_{2}=35 \times 10^{-3} \mathrm{ps}^{2} / \mathrm{m}, \sigma=6 \times 10^{-3}(\mathrm{~W} \mathrm{~m})^{-1}$ and $g=1.44 \mathrm{~m}^{-1},{ }^{3}$ we have $\epsilon \sim 0.04$.

\section{PERTURBATION ANALYSIS}

In this section we exploit the small parameter $\epsilon$ in Eq. (2), and use a perturbation analysis to obtain the leading order modulations to the amplitude and phase of the similariton pulse induced by TOD. Equation (2) has non-constant coefficients due to their dependence on $\tau$. It is easy to show that assuming

$$
\eta \ll \frac{3 \sigma \tau_{0}^{2}}{2 \epsilon^{2} g} \equiv \eta^{*} \Leftrightarrow z \ll \frac{3 \ln 2}{2 g} \equiv z^{*},
$$

allows us to expand $\tau(\eta)$ about $\tau_{0}$ and obtain $\tau(\eta)=\tau_{0}+\epsilon^{2} g \eta /\left(3 \sigma \tau_{0}\right)+O\left(\epsilon^{4}\right)$. We see that condition (4) imposes a limit on the propagation distances where our model holds. For example, $\eta^{*} \simeq 33$ or $z^{*} \simeq 0.72 \mathrm{~m}$ for the fibre parameters used in Sec. 2. However, the underlying TOD dynamics occurs within distances $z \sim z^{*}$ for a wide variety of parameter values.

Now, looking for a solution of Eq. (2) of the form: $f(\eta, \xi)=A(\eta, \xi) \exp [i \phi(\eta, \xi)]$ $=\sum_{j=0}^{\infty} \epsilon^{j} A_{j}(\eta, \xi) \exp \left[i \sum_{j=0}^{\infty} \epsilon^{j} \phi_{j}(\eta, \xi)\right]$, we find the leading order solution is given by the similariton solution: ${ }^{6}$

$$
A_{0}(\xi)=\sqrt{\lambda\left(1-\xi^{2}\right)}, \quad \phi_{0}(\eta)=\lambda \eta .
$$

Note that for this solution, the parameter $\lambda=1$. The first non-zero perturbation comes at $O\left(\epsilon^{2}\right)$, where we obtain the solution

$$
\begin{array}{rlrl}
A_{2}(\xi, \eta) & =R(\xi) \eta, & \phi_{2}(\xi, \eta) & =R(\xi) A_{0}(\xi) \eta^{2}+G(\xi) \eta \\
R(\xi)=\frac{\beta_{3}}{6 \sigma}\left[\frac{1}{\tau_{0}^{5}} A_{0 \xi \xi \xi}-\frac{12 C^{2}}{\tau_{0}} \xi\left(\xi A_{0}\right)_{\xi}\right], & G(\xi) & =-\frac{\beta_{2}}{2 \sigma \tau_{0}^{4}} \frac{A_{0 \xi \xi}}{A_{0}}+\frac{\beta_{3}}{6 \sigma}\left[\frac{6 C}{\tau_{0}^{3}} \frac{\left(\xi A_{0 \xi}\right)_{\xi}}{A_{0}}-8 C^{3} \tau_{0} \xi^{3}\right] .
\end{array}
$$

Note that the coefficient functions $R(\xi)$ and $G(\xi)$ are dependent on the leading order solution, while the amplitude (phase) perturbation is linear (quadratic) in $\eta$. Further, in the absence of TOD there is no amplitude modulation 
and a symmetric (in $\xi$ ) phase perturbation. The latter comes from the semi-classical approximation that one can apply to the unperturbed form of Eq. (1) to obtain the self-simlar solution (5). ${ }^{6}$ Functions $R(\xi)$ and $G(\xi)$ are singular at the points $|\xi|=1$ where the purely parabolic solution $A_{0}$ in (5) vanishes. To remove this problem, here we use a particular approximation for $A_{0},{ }^{18}$ which coincides with the parabolic pulse shape in the central core and, at the same time, approximates the smooth decrease of the amplitude in the pulse tails which is observed in the true asymptotic pulse solution. A parameter $\delta$, with $0<\delta<1$, parametrizes the size of the pulse central region, and can be adjusted to achieve a good approximation for the particular pulse properties to be described.

The perturbation expansion can be carried out to all orders, however for the purpose of this paper we will only consider the leading order perturbation. Although the expressions (7) are cumbersome, analytical insight can be gained. Specifically, it is interesting that the intensity and phase perturbations can be approximated by cubic polynomials in the variable $\xi$ :

$$
A^{2}-A_{0}^{2} \simeq \frac{4 \epsilon^{2} \beta_{3} C^{2} \eta}{\sigma \tau_{0}}\left(2 \xi^{3}-\xi\right)+O\left(\epsilon^{4}\right), \quad \phi-\phi_{0} \simeq \frac{2 \epsilon^{2} \beta_{3} C^{2} \eta}{\sigma}\left[\frac{\eta}{\tau_{0}}\left(2 \xi^{3}-\xi\right)-\frac{2 C \tau_{0}}{3} \xi^{3}\right]
$$

in the region $|\xi| \leq 1-\delta$. In addition to the pulse perturbations (6), we can calculate the skewness $S$, which is typically used to quantify the asymmetry of the pulse temporal shape, as

$$
S \equiv \int \xi^{3} A^{2} \mathrm{~d} \xi=\frac{\epsilon^{2} \beta_{3}}{\sigma}\left[\frac{1}{\tau_{0}^{5}} f_{1}(\delta)+\frac{C^{2}}{\tau_{0}} f_{2}(\delta)\right] \eta,
$$

where the functions $f_{1}$ and $f_{2}$ can easily be calculated as a function of $\delta$.

\section{COMPARISON THEORY VS. NUMERICAL SIMULATIONS}

In this section we compare the predictions from the analytic model developed with the results of numerical integration of Eq. (1). We numerically generate a parabolic pulse through the reshaping of a Gaussian pulse which occurs upon propagation in a section of fibre with the SOD, nonlinearity and gain parameters mentioned in Sec. 2. The formed pulse is used as the initial pulse in our study, and propagated over the distance $z=z^{*}$ in the gain fibre with the TOD switched on.

Figure 1 shows the temporal distributions of the perturbations in the pulse intensity and phase plotted in $z /\left(4 z^{*}\right)$ increments along the fibre, as obtained from NLS numerical simulation and Eqs (5)-(7) for the TOD parameter $\beta_{3}=0.7 \times 10^{-3} \mathrm{ps}^{3} / \mathrm{m}$. The simulation results are in good agreement with the analytic results in the central portion of the pulse containing most of the pulse energy, even at propagation distances $z \sim z^{*}$ where condition (4) is not satisfied. Also plotted in Fig. 1 are the cubic polynomials (8), and show that they are an excellent approximation to the overall pulse perturbation due to the TOD. Equations (8) thus provide simple analytic expressions to describe the changes induced by TOD on the temporal intensity and phase distributions of the pulse. The pulse temporal intensity and chirp profiles are plotted in Fig. 2 at the same points along the fibre as in Fig. 1. We see that the TOD makes the pulse shape become asymmetric with the peak shifted to the leading edge, corresponding to a red-shifted spectrum. As the propagation distance increases, the asymmetry becomes stronger. Further, Fig. 2 highlights the approximatively parabolic nature of the chirp induced by TOD in the central region of the pulse. Note that $\phi_{t}=(\arg U)_{t}-2 C t$ and, therefore, the pulse chirp should ideally be zero in the absence of TOD.

In Fig. 3 the skew parameter $S$ from both numerical simulation of Eq. (1) and the theoretical prediction from Eq. (9) is shown for various $\beta_{3}$ values and propagation distances. The skew parameter from numerical simulation increases linearly with both the TOD values and propagation distance up to the onset of pulse break-up. Equation (9) predicts a linear increase of the skew parameter with $\beta_{3}$ and an linear increase with the self-similar variable $\eta$. However, a linear dependence in $\eta$ is equivalent to an exponential dependence in propagation distance $z$. This can be accounted for by the fact that our perturbation analysis is valid only for $z<z^{*}$, which corresponds to a nearly linear relationship between $z$ and $\eta$ in the propagation region considered. Figure 3 shows reasonable agreement between the analytic model and the simulation results in the parameter region preceeding wave breaking with

relative error up to $\sim 16$ percent. In all simulations performed we find that the critical value of the skewness for 

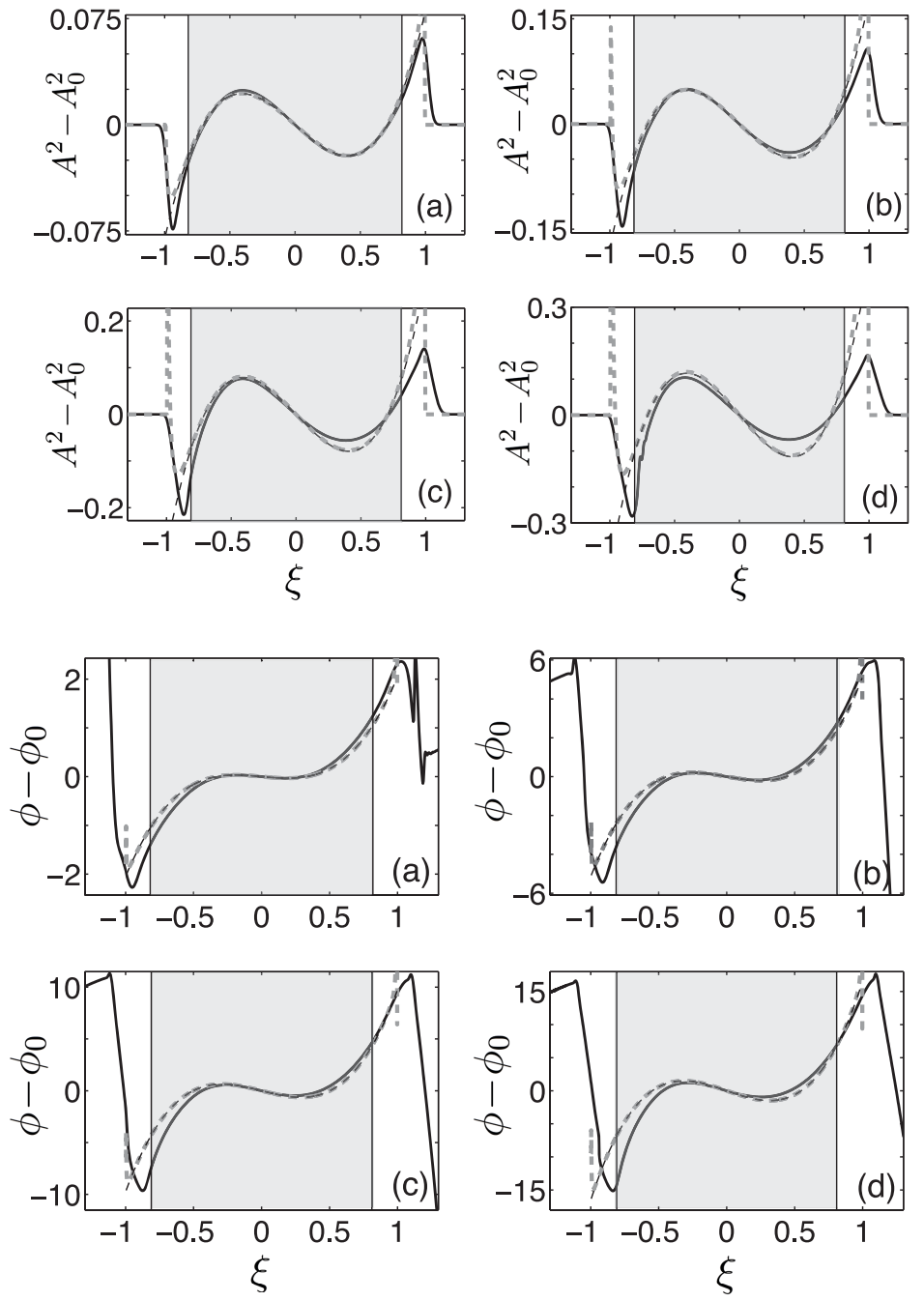

Figure 1. Perturbation of the pulse intensity (top) and phase (bottom) from NLS numerical simulation (solid black) compared to the theoretical prediction (dashed grey) at four points along the fibre: $z / z^{*}=(\mathrm{a}) 0.25$ (b) 0.5 (c) 0.75 and (d) 1. Also shown are the approximate cubic polynomials (dashed black). The shaded region corresponds to 95 percent of the pulse energy. 

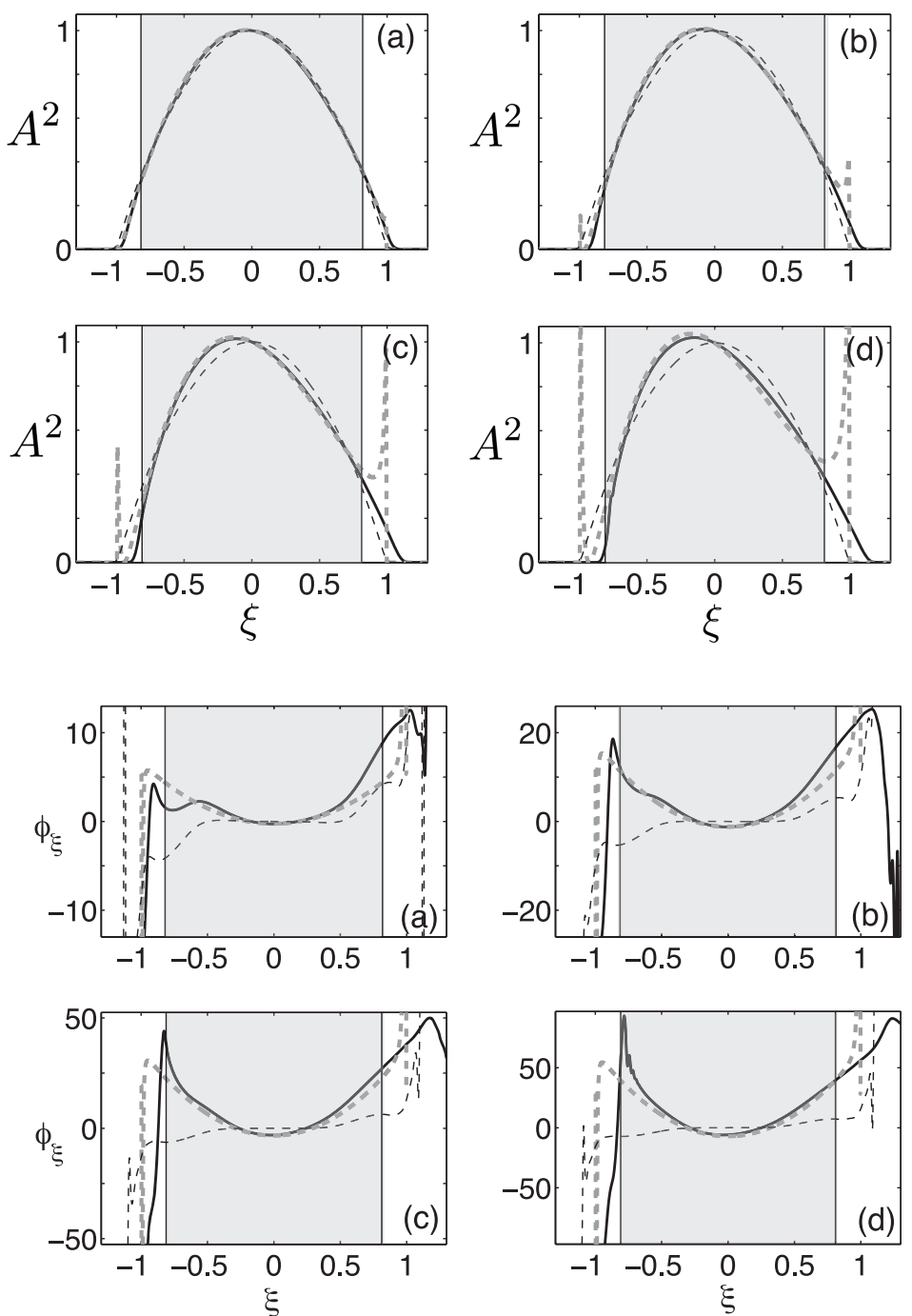

Figure 2. Pulse intensity profile (top) and chirp profile (bottom) from NLS numerical simulation (solid black) compared to the theoretical prediction (dashed grey) at four points along the fibre: $z / z^{*}=$ (a) 0.25 (b) 0.5 (c) 0.75 and (d) 1. The pulse solution with no TOD effect is also shown (dashed black). The shaded region corresponds to 95 percent of the pulse energy. 

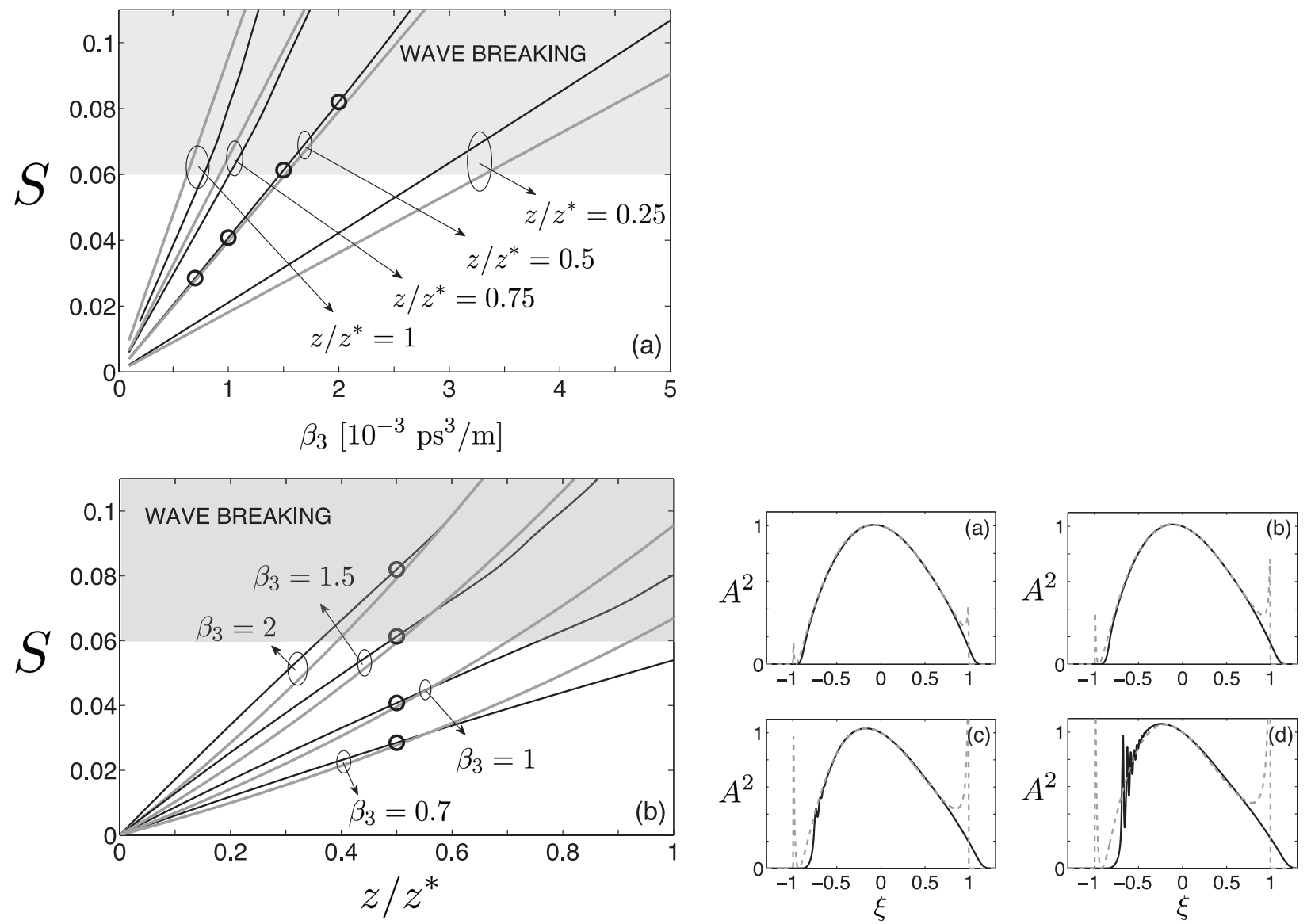

Figure 3. Left: Skew parameter $S$ from NLS numerical simulation (black) as well as the theoretical prediction (grey) as a function of (a) $\beta_{3}$ at four points along the fibre and (b) $z / z^{*}$ for four values of $\beta_{3}$ (in $10^{-3} \mathrm{ps}^{3} / \mathrm{m}$ ). The bold circles correspond to common points of $(\mathrm{a})$ and $(\mathrm{b})$. Wave breaking occurs when $S \sim 0.06$. Right: Pulse temporal intensity profiles from NLS numerical simulation (solid) as well as the theoretical prediction (dashed) for four values of the skew parameter: $S \approx$ (a) 0.03 (b) 0.04 (c) 0.06 and (d) 0.08 . The profiles are taken at the bold circled points of the left graph.

which a shock-wave-type of instability starts to develop on the pulse due to the TOD is $S \sim 0.06$. Also shown in Fig. 3 are the pulse temporal intensity profiles taken at different values of the skew parameter. Note that although the theoretical prediction of the pulse perturbation (Eq. (6)) cannot predict the TOD-induced shock in the leading edge of the pulse, it is capable of predicting the form of the pulse with sufficient accuracy even after the onset of the shock.

\section{CONCLUSION}

We have found the leading order structural changes induced by TOD on the similariton pulse solution of the NLS equation in a fibre with constant linear gain. Analytic expressions for the perturbations of the temporal amplitude and phase of the pulse as well as the pulse skewness have been derived. We have found that the effects of the TOD on both the pulse shape and phase can be well approximated by cubic polynomials in the temporal variable. The analytic results have been confirmed by extensive numerical simulations of the governing equation.

\section{ACKNOWLEDGMENTS}

This research was supported by the Engineering and Physical Sciences Research Council (grant EP/F02956X/1). 


\section{REFERENCES}

[1] Anderson, D., Desaix, M., Karlsson, M., Lisak, M., and Quiroga-Teixeiro, M. L., "Wave-breaking-free pulses in nonlinear-optical fibers," J. Opt. Soc. Am. B 10, 1185-1190 (1993).

[2] Dudley, J. M., Finot, C., Millot, G., and Richardson, D. J., "Self-similarity and scaling phenomena in ultrafast nonlinear optics," Nat. Phys. 3, 597-603 (2007).

[3] Fermann, M. E., Kruglov, V. I., Thomsen, B. C., Dudley, J. M., and Harvey, J. D., "Self-similar propagation and amplification of parabolic pulses in optical fibers," Phys. Rev. Lett. 84, 6010-6013 (2000).

[4] Kruglov, V. I., Peacock, A. C., Dudley, J. M., and Harvey, J. D., "Self-similar propagation of high power parabolic pulses in optical fiber amplifiers," Opt. Lett. 25, 1753-1755 (2000).

[5] Kruglov, V. I., Peacock, A. C., Harvey, J. D., and Dudley, J. M., "Self-similar propagation of parabolic pulses in normal dispersion fiber amplifiers," J. Opt. Soc. Am. B 19, 461-469 (2002).

[6] Boscolo, S., Turitsyn, S. K., Novokshenov, V. Y., and Nijhof, J. H. B., "Self-similar parabolic optical solitary waves," Theor. Math. Phys. 133, 1647-1656 (2002).

[7] Kruglov, V. I., and Harvey, J. D., "Asymptotically exact parabolic solutions of the generalized nonlinear Schrödinger equation with varying parameters," J. Opt. Soc. Am. B 23, 2541-2550 (2006).

[8] Ilday, F. Ö., Buckley, J., Wise, F. W., and Clark, W. G., "Self-similar evolution of parabolic pulses in a laser," Phys. Rev. Lett. 92, 213902(4) (2004).

[9] Hirooka, T., and Nakazawa, M., "Parabolic pulse generation by use of a dispersion-decreasing fiber with normal group velocity dispersion," Opt. Lett. 29, 498-500 (2004).

[10] Shah, L., Liu, Z., Hartl, I., Imeshev, G., Cho, G. C., and Fermann, M. E., "High energy femtosecond Yb cubicon fiber amplifier," Opt. Express 13, 4717-4722 (2005).

[11] Zhou, S., Kuznetsova, L., Chong, A., and Wise, F. W., "Compensation of nonlinear phase shifts with third-order dispersion in short-pulse fiber amplifiers," Opt. Express 13, 4869-4877 (2005).

[12] Logvin, Y., Kalosha, V. P., and Anis, H., "Third-order dispersion impact on mode-locking regimes of Ybdoped fiber laser with photonic bandgap fiber for dispersion compensation," Opt. Express 15, 985-991 (2007).

[13] Ruehl, A., Prochnow, O., Schultz, M., Wandt, D., and Kracht, D., "Impact of third-order dispersion on the generation of wave-breaking free pulses in ultrafast fiber lasers," Opt. Lett. 32, 2590-2592 (2007).

[14] Latkin, A. I., Turitsyn, S. K., and Sysoliatin, A. A., "Theory of parabolic pulse generation in tapered fiber," Opt. Lett. 32, 331-333 (2007).

[15] Wabnitz, S., and Finot, C., "Theory of parabolic pulse propagation in nonlinear dispersion-decreasing optical fiber amplifiers," J. Opt. Soc. Am. B 25, 614-621 (2008).

[16] Bale, B. G., and Boscolo, S., "Impact of third-order fibre dispersion on the evolution of parabolic optical puses," J. Opt. 12, 015202(6) (2010).

[17] Agrawal, G. P., [Nonlinear Fiber Optics], Academic Press (2001 (third edition)).

[18] Turitsyn, S. K., and Boscolo, S., "Dissipative nonlinear structures in fiber optics," in [Dissipative Solitons: From Optics to Biology and Medecine] (Lecture Notes in Physics vol. 751), Akhmediev, N., and Ankiewicz, A., eds., 195-220, Heidelberg, Springer (2008). 